\title{
A Poorly Prognostic Case of Renal Dialysis in a 20- Year Case Series of Adult Coronary Sinus Atrial Septal Defect Repair at New Tokyo Hospital
}

\author{
Tatsuya Nakao \\ New Tokyo Hospital \\ Yuki Ikegaya \\ New Tokyo Hospital \\ Daisuke Iwahashi \\ New TokyoHospital \\ Shoich Tsuda \\ New Tokyo Hospital \\ Nao Kume \\ New Tokyo Hospital \\ Sunao Nakamura \\ New Tokyo Hospital
}

Haruhiko Sugimori ( $\sim$ haru-sugimori@mvc.biglobe.ne.jp )

New Tokyo Hospital https://orcid.org/0000-0002-9920-119X

\section{Case report}

Keywords: Coronary sinus atrial septal defect, Unroofed coronary sinus syndrome, Poor prognosis, Renal dialysis, Adult congenital heart disease

Posted Date: December 15th, 2020

DOI: https://doi.org/10.21203/rs.3.rs-127860/v1

License: (c) (1) This work is licensed under a Creative Commons Attribution 4.0 International License. Read Full License 


\section{Abstract}

Background: Isolated coronary sinus atrial septal defect (ASD) is defined as a coronary sinus unroofed in the terminal portion without a persistent left superior vena cava and other anomalies. This defect is rare and part of a wide spectrum of unroofed coronary sinus syndromes. Recently, several reports have described this finding. We searched the hospital's database to determine the incidence of this defect, and to raise awareness of this condition, we discussed the findings from five patients with coronary sinus ASD who underwent surgical repair.

Case presentation: The patients were three women and two men with an age range of 63-77 years. All patients underwent transthoracic echocardiography and computed tomography, and one underwent magnetic resonance imaging. In two patients, the defect was found unexpectedly intraoperatively; left-toright shunting was apparent in the other three patients preoperatively. The pulmonary-to-systemic blood flow ratio ranged from 1.42 to 3.1 following cardiac catheterization, and oxygen saturation step-up was seen on the right side of the heart. Valvular regurgitation was seen in 4/5 patients with the mitral, tricuspid, and aortic valves involved in different combinations and to different degrees. Right atrial and ventricular dilation were seen in 4/5 patients; three patients had left atrial dilation. Three patients experienced atrial fibrillation, and one of these also experienced paroxysmal ventricular contractions. All patients underwent surgical repair, and some underwent multiple procedures. One patient who had previously undergone kidney transplantation died approximately 1 year postoperatively; the remaining four patients are currently experiencing good activities of daily living without symptoms.

Conclusions: Coronary sinus ASD (Kirklin and Barratt-Boyes type IV unroofed coronary sinus syndrome) comprised $1.3 \%$ of adult congenital heart surgeries and $0.07 \%$ of adult open-heart surgeries in our hospital from 1999 to 2019. Our hospital performs cardiac surgery mainly for patients with acquired cardiac disease, and coronary sinus ASD is rare. Early diagnosis and, in symptomatic patients (especially those with blood access shunts, which may overload the heart), early surgical repair are important. The poorly prognostic case in our series is noteworthy, as similar cases have not been reported previously.

\section{Full Text}

Due to technical limitations, full-text HTML conversion of this manuscript could not be completed. However, the latest manuscript can be downloaded and accessed as a PDF.

\section{Tables}

Due to technical limitations, table 1 to 5 is only available as a download in the Supplemental Files section.

\section{Figures}



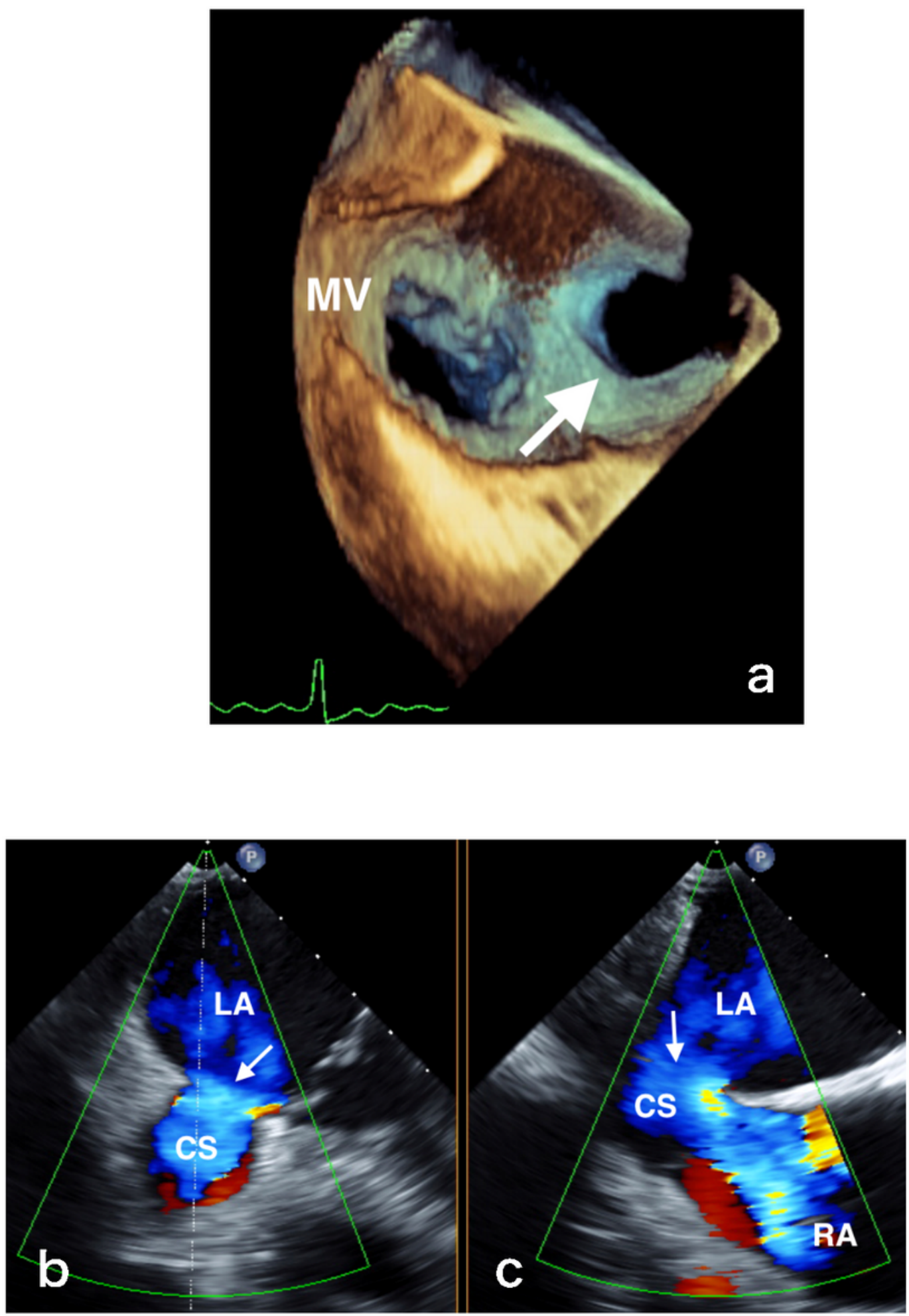

\section{Figure 1}

Echocardiographic findings in cases 3 and 5 a. Three-dimensional transesophageal echocardiography in case 5 clearly showing a defect (white arrow) near the posterolateral commissure of the mitral valve (MV). b, c. Dynamic left-to-right shunting is visible through the defect in case 3. CS coronary sinus, LA left atrium, MV mitral valve, RA right atrium 


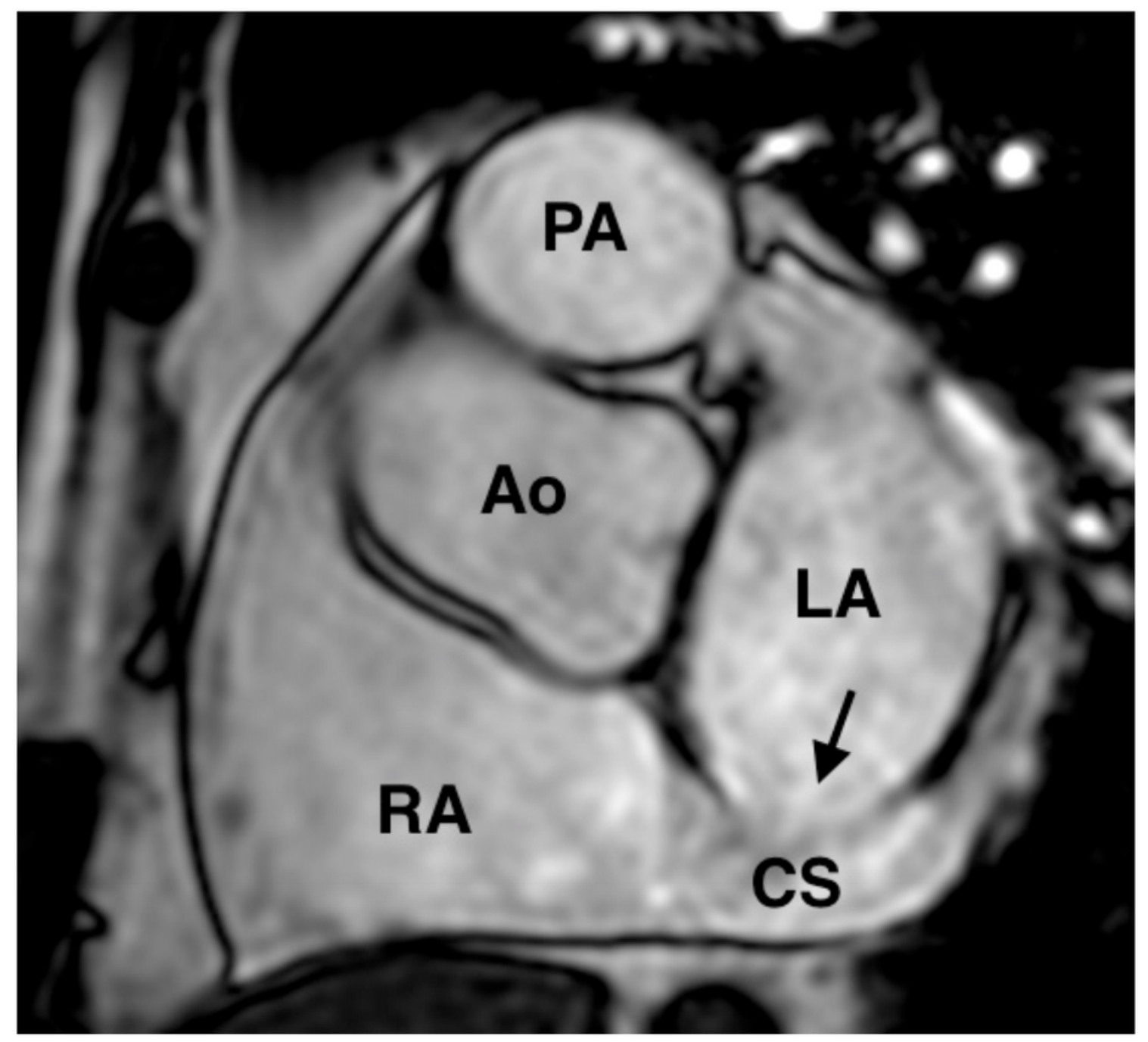

\section{Figure 2}

Magnetic resonance imaging in case 4 Magnetic resonance imaging (sagittal view) in case 4 showing the morphology of the patient's congenital heart disease. We found no persistent left superior vena cava or other congenital cardiovascular anomalies. The arrow indicates the coronary sinus defect. Ao aorta, CS coronary sinus, LA left atrium, PA pulmonary artery, RA right atrium 


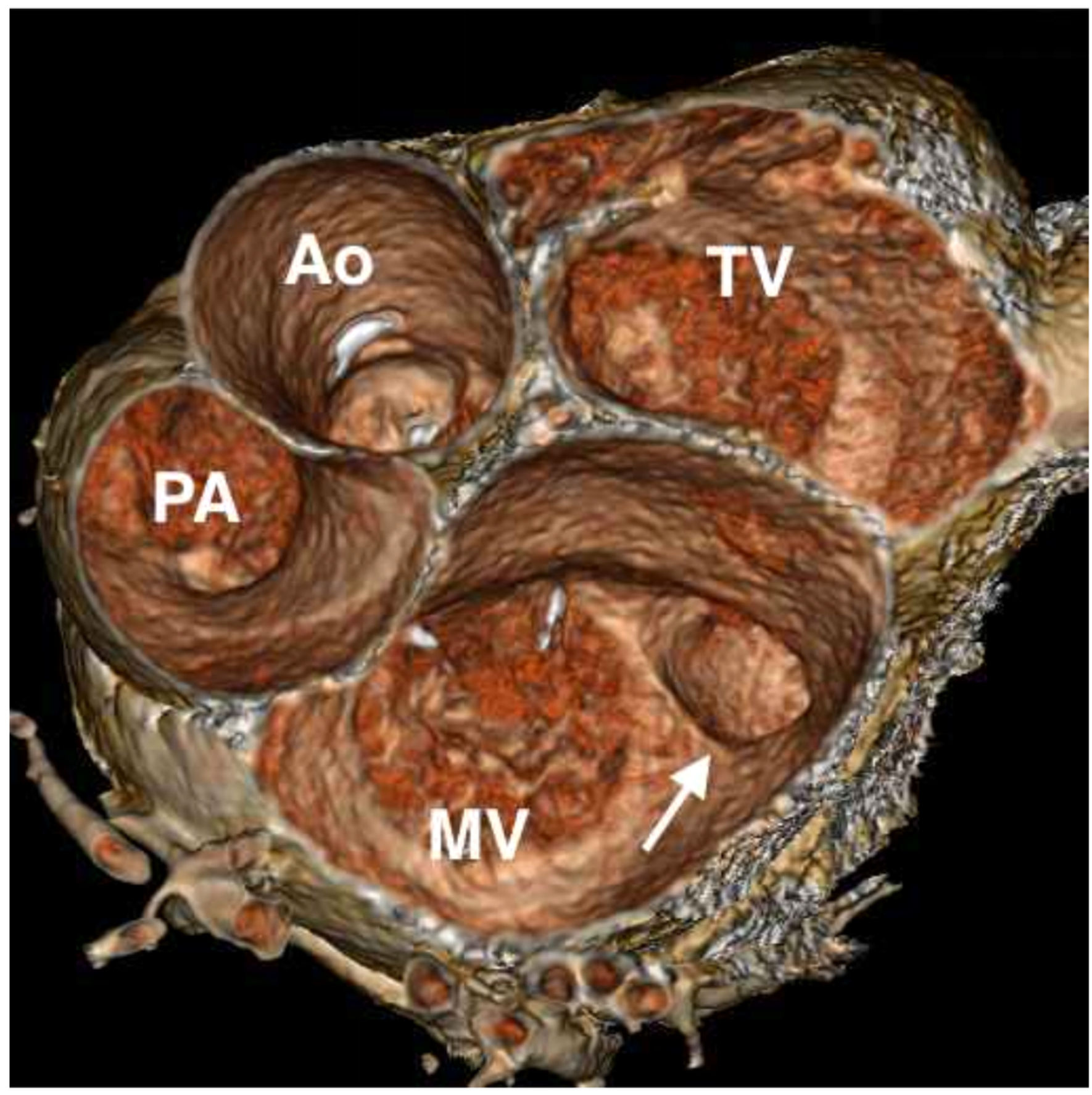

\section{Figure 3}

Sixty-four-row multidetector computed tomography scan in case 5 Sixty-four-row multidetector computed tomography scan in case 5 showing the morphology of the patient's congenital heart disease. The arrow indicates the coronary sinus defect. Ao aorta, MV mitral valve, PA pulmonary artery, TV tricuspid valve 


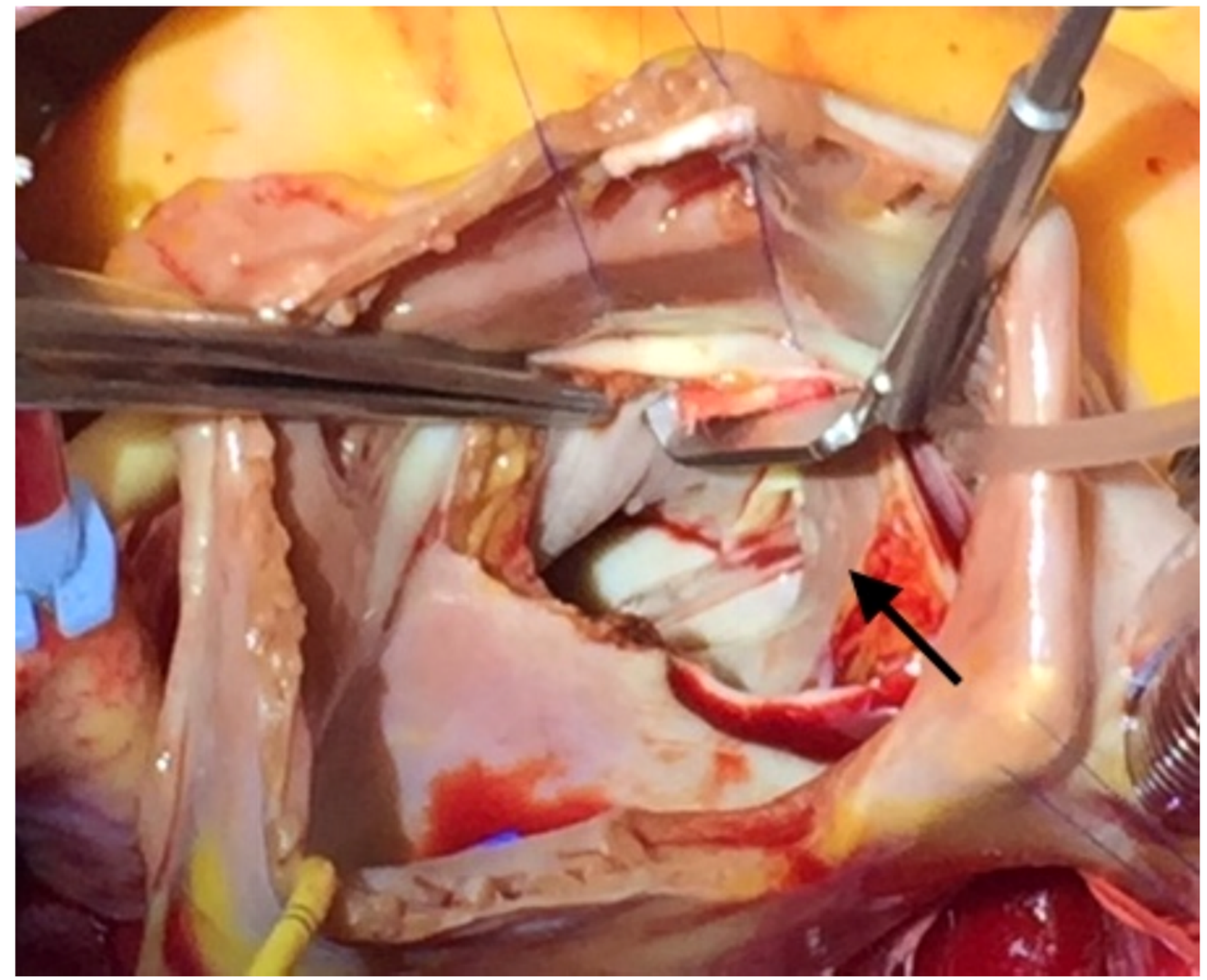

Figure 4

Coronary sinus defect in case 3 We confirmed communication between both atria through the defect (arrow) by inserting a catheter through the coronary sinus orifice. The image is a surgical photograph from case 3 .

\section{Supplementary Files}

This is a list of supplementary files associated with this preprint. Click to download.

- CSASDTablesJCS.pdf

- CSASDsuppletable1JCS.pdf 